\title{
Optimization of the Natural Gas Purification Process Based on Exergy Analysis
}

\author{
Baocheng Shi $(\mathbb{D}$, Jianpeng Pan, Xiaochi Guo, Xingkai Zhang, Lijuan Wu, Kai Liu, \\ and Yindi Zhang
}

School of Petroleum Engineering, Yangtze University, Wuhan 430100, China

Correspondence should be addressed to Baocheng Shi; shibaoch1@163.com

Received 11 August 2020; Revised 18 September 2020; Accepted 27 October 2020; Published 29 November 2020

Academic Editor: Guanglong Sheng

Copyright (c) 2020 Baocheng Shi et al. This is an open access article distributed under the Creative Commons Attribution License, which permits unrestricted use, distribution, and reproduction in any medium, provided the original work is properly cited.

\begin{abstract}
This study is aimed at carrying out investigations on a domestic gas field, located in Yanchang, China, with a view to optimize the natural gas purification process. The main objectives of this work are (i) to reduce the natural gas purification system's energy consumption and (ii) improve the existing purification levels. Process simulations were carried out using Aspen Plus ${ }^{\mathrm{TM}}$ software, and a comprehensive technical and economic analysis was carried out. The single-factor sensitivity analysis method was used to determine the parameters of absorption, such as the reflux ratio and number of stages. The heat transfer process was analyzed using the energy-saving method of the energy system, and a modified process was recommended. The optimization results show that the recommended system has better purification performance, the comprehensive energy consumption is effectively reduced, and the energy efficiency is improved by $9 \%$.
\end{abstract}

\section{Introduction}

To promote a healthy, sustainable, and stable development of our national economy, the energy requirements of highenergy-consuming systems should be reduced. Therefore, improving the energy utilization rate has become one of the main research directions. In the field of natural gas purification, high-energy consumption and complex processes are widely prevalent [1], which makes it a potential candidate for carrying out an energy-saving analysis and reducing the energy consumption. Most of the studies carried out in the past only analyzed the process flow from the perspective of a single aspect, which usually resulted in a limited degree of optimization. Thus, the optimization results were inadequate to meet the needs of the industry, and the benefits of the transformation were not obvious. Based on a summary of the optimization schemes employed in the previous works, a multidimensional optimization and a comprehensive and systematic analysis of natural gas purification systems have been conducted in this study from the perspectives of process and technology. This study was conducted at the Yanchang gas field in China. The first step involved the selection of a deacidification reagent. The current solution systems include methyl diethanolamine (MDEA) solution, MDEA complex solution, activated MDEA solution, MDEA-sulfolane solution, and MDEA mixed solution. The second step involved the improvement of the deacidification process, for which there were two options, the poor/rich liquid circulation system and the semipoor liquid circulation system. The third step involved the modification of the dehydration process through low-temperature separation, solvent absorption, solid absorption, supersonic dehydration, and membrane separation. The final step involved the optimization of the heat exchanger network from the perspective of exergy analysis, which not only improves energy efficiency but also saves energy.

\section{Optimization of the Natural Gas Purification Process Based on Exergy Analysis}

\subsection{Deacidification Process Optimization}


2.1.1. Reagent Selection. The common acid gas components in the natural gas industry are $\mathrm{CO}_{2}$ and $\mathrm{H}_{2} \mathrm{~S}$. For meeting a variety of requirements, such as environmental protection from exhaust gas emissions, protection of equipment and pipelines from corrosion, and downstream processes, $\mathrm{H}_{2} \mathrm{~S}$ gas must be removed from the systems. It should be noted that the requirements for the allowable $\mathrm{H}_{2} \mathrm{~S}$ content are very stringent. However, $\mathrm{CO}_{2}$ is mainly responsible for greenhouse gases, which have serious consequences for the environment. $\mathrm{CO}_{2}$ recovery and emission reduction are the main methods to mitigate the greenhouse effect [2]. Macroscopically speaking, acid gas deacidification can be divided into two methods, chemical absorption and physical adsorption. The sulfur part of the Sulige gas field is located in the Lower Paleozoic horizon, mainly Taiyuan Formation and Mawu Formation, while the gas well horizon of the Yanchang oilfield includes Benxi Formation, Shanxi Formation, and Shihezi Formation of Upper Paleozoic, so it does not contain sulfur and is absent in the natural gas processing components as well but has relatively low gas source pressure and high-water content. Therefore, it has higher treatment requirements in the downstream treatment. Hence, the only other main component of acid gas is $\mathrm{CO}_{2}$. The acid gas of the original treatment plant was removed by the chemical absorption method. The reagent used was MDEA solution, i.e., aqueous N-methyl diethanolamine. MDEA is a tertiary amine, and its deacidification mechanism is shown in formulas (1)-(3) [3-5].

$$
\begin{gathered}
\mathrm{CO}_{2}+\mathrm{R}_{2} \mathrm{NCH}_{3} \text { (nonreactive) } \\
\mathrm{CO}_{2}+\mathrm{H}_{2} \mathrm{O} \longrightarrow \mathrm{H}^{+}+\mathrm{HCO}_{3}{ }^{-} \quad \text { (long response) } \\
\mathrm{H}^{+}+\mathrm{R}_{2} \mathrm{NCH}_{3} \longrightarrow \mathrm{R}_{2} \mathrm{NCH}_{3} \mathrm{H}^{+} \quad \text { (long response) }
\end{gathered}
$$

MDEA is a reagent for selective absorption of sulfur in natural gas purification pretreatment in use since the early 1980s. It has many advantages, such as good degradation performance, great chemical stability, long shelf life, low circulation of amine liquid, and relatively low price [6]. However, MDEA foams more easily than the other amine liquids and, hence, can often lead to false liquid levels in the absorption and regeneration towers. Furthermore, it is not conducive for personnel to operate and manage the process and can, therefore, even cause serious safety issues. To improve the stability of the MDEA system and comply with the principle of selecting reagents according to the $\mathrm{CO}_{2}$ concentration in engineering [7], two suitable optimization schemes are proposed for this system:

(1) The compound amine solution is used for deacidification. Compound amine solution has many advantages, such as ability to improve purification, high absorption load capability, capacity for fast absorption of the acid gas, lower levels of corrosion of equipment and pipelines, and low energy consumption of regeneration and low evaporation loss. It makes up for the shortcomings of the single amine solution $[8,9]$. The compounding scheme used in this study is based on the previous literature [10], with a specific combination of $2 \mathrm{~mol} / \mathrm{L}$ MDEA with sulfolane in a ratio of $10: 3$ to create a complex amine liquid

(2) Because there is no active $\mathrm{H}$ atom in the MDEA molecule, its reaction must be based on the reaction of $\mathrm{CO}_{2}$ with $\mathrm{H}_{2} \mathrm{O}$ (control step) [11]. Thus, its absorption of $\mathrm{CO}_{2}$ can be improved by activating the MDEA method [12]. By adding primary amine, secondary amine, or another activator to the MDEA solution, this method can achieve high acid gas load and low energy consumption. Taking primary amine as an example, the reaction mechanism is shown in (4)-(6) [13]:

$\mathrm{CO}_{2}+2 \mathrm{RNH} \longrightarrow \mathrm{RNHCOO}^{-}+\mathrm{RNH}_{2}^{+}$(medium speed reaction $)$

$\mathrm{CO}_{2}+\mathrm{RNH}+\mathrm{H}_{2} \mathrm{O} \longrightarrow \mathrm{RNH}_{2}^{+}+\mathrm{HCO}_{3}{ }^{-} \quad$ (long response)

$$
\begin{aligned}
\mathrm{RNHCOOH} & +\mathrm{RCH}_{3} \mathrm{NH}+\mathrm{H}_{2} \mathrm{O} \longrightarrow \mathrm{RNH} \\
& +\mathrm{RCH}_{3} \mathrm{NH}^{+} \cdot \mathrm{HCO}_{3}{ }^{-}
\end{aligned}
$$

The abovementioned reactions show that the activator forms carboxylic acid after absorbing $\mathrm{CO}_{2}$ and then immediately transfers it to the liquid phase to form bicarbonate. In this process, the activator acts as a catalyst without consumption [14]. Therefore, an activator is very commonly used in natural gas decarbonization treatment. Using the experience of the past studies $[15,16]$, in this study, PZ (piperazine) with a superior activation performance is used as the activator. This activator has strong adaptability and can meet the requirements of $\mathrm{CO}_{2}$ absorption at different concentrations. This is because in the later stage of the gas field exploitation, as the availability of the natural gas reserves decreases, it is accompanied by an increase in the $\mathrm{CO}_{2}$ content. Therefore, the selection of an activator with a wide range of sensitivity has a positive significance for gas treatment in the later stage of the gas field. The mass fraction of PZ in the final selected activated MDEA solution is $3 \%-5 \%$.

2.1.2. Process Improvement. By studying the influence of several process parameters on the $\mathrm{CO}_{2}$ content in the purified gas and the operational cost of the unit, it is concluded that the absorption temperature of the amine solution, circulation volume, and regeneration tower temperature all have a significant influence on the operational cost of the unit [17]. Of these three, the most influential factors are the circulating quantity of the amine solution and the regeneration temperature. A small change in these two parameters would cause the operational cost of the unit to increase significantly. Thus, the superiority of the semilean solution scheme [18] (wherein half of the total circulating solution is extracted from the middle of the regeneration tower, after passing through the semilean liquid/rich liquid heat transfer, and is reintroduced to the middle of the absorption tower) can be evident. 
2.2. Dehydration Process Optimization. In addition to solid and acid gases, impurities in raw natural gas also contain water. The existence of water increases the risk of hydrate formation, causes ice blockage of pipelines and equipment, affects the calorific value of the natural gas, reduces the quality of natural gas, and wastes energy in the downstream treatment [19]. Therefore, dehydration of natural gas is very essential. There are two methods for water treatment, chemical absorption and physical absorption [20] (membrane separation [21, 22], silica gel and molecular sieve method [23]). To improve the recovery of natural gas in the later stage of gas field exploitation, pressurized foam drainage or fracturing technology is generally adopted, which will inevitably lead other pollutants such as reagents, oil pollution, and gas field water into the purification process, thus affecting the dehydration effect. Secondly, the increase of turbocharging will cause airflow pulsation, which will bring great hidden dangers to the safe production of natural gas purification. Moreover, the consumption of triethylene glycol (TEG) in the TEG dehydration process will account for more than half of the dehydration process and become an important factor affecting the operation cost of the dehydration process [24]. At the same time, considering that most of the natural gas output of the Yanchang gas field will be sent to an LNG processing plant, a three-tower molecular sieve dewatering process is recommended to meet the high standards of the LNG gas source and the relevant requirements of storage and transportation in the health, safety, and environment (HSE) management system $[25,26]$. A typical three-tower molecular sieve dehydration process is available in the literature [27]. The shadow part shows that the water content is basically saturated, and the blank area shows that water can also be adsorbed.

2.3. Exergy Analysis. The first law of thermodynamics states that energy is conserved when it is transformed or transferred, and the second law of thermodynamics states the degree and direction of the process. Exergy analysis combines the first and second laws. It is a scientific energy analysis method of energy systems. It plays a unique role in the global energy-saving practice $[28,29]$. Exergy refers to the maximum functional power [30] when the system is in a certain state and can be expressed as

$$
E_{x}=\left(H-H_{0}\right)-T_{0}\left(S-S_{0}\right),
$$

where $T_{0}$ is the ambient temperature, ${ }^{\circ} \mathrm{C} ; H$ is the specific enthalpy, $\mathrm{kJ} ; H_{0}$ is the specific enthalpy under ambient temperature, kJ; $S$ is the entropy, kJ; and $S_{0}$ is the entropy under ambient temperature, $\mathrm{kJ}$.

In recent decades, the modern energy use theory, which takes the method of exergy analysis as the core, has been widely used in energy management, petrochemical industry, thermal power plants, refrigeration technology, and other fields. It provides a scientific basis for further improving the level of energy use and effectively reducing the energy consumption index. There are mainly three kinds of exergy analysis models [31]. Generally, the black box and gray box models are mostly used for qualitative analysis of the energy
TABLE 1: Components of natural gas.

\begin{tabular}{lccc}
\hline Components & $\begin{array}{c}\text { Mole percent } \\
(\%)\end{array}$ & Components & $\begin{array}{c}\text { Mole percent } \\
(\%)\end{array}$ \\
\hline $\mathrm{He}$ & 0.0314 & $\mathrm{CH}_{3}-\mathrm{CH}_{2}-\mathrm{CH}_{3}$ & 0.0214 \\
$\mathrm{H}_{2}$ & 0.0152 & $\mathrm{CH}_{3}-\mathrm{CH}_{-}-\mathrm{CH}_{3}$ & 0.0015 \\
$\mathrm{~N}_{2}$ & 0.2698 & $\mathrm{CH}_{3}-\mathrm{CH}_{2}-\mathrm{CH}_{2}-$ & 0.0023 \\
$\mathrm{CO}_{2}$ & 2.9078 & $\mathrm{CH}_{3}$ & 0.0005 \\
$\mathrm{CH}_{4}$ & 96.105 & $\left(\mathrm{CH}_{3}\right)_{4}$ & $\mathrm{CHCH}_{2} \mathrm{CH}_{3}$ \\
$\mathrm{CH}_{3}-\mathrm{CH}_{3}$ & 0.5676 & $\mathrm{Hg}$ & 0.0006 \\
\hline
\end{tabular}

consumption system, and then the fuzzy analysis results are used to carry out energy-saving transformation. However, the energy consumption data of each unit of the system is complete, and the white box model analysis can ensure the accuracy of the data, so as to obtain a better transformation effect, so the white box model is used in this paper.

2.4. Heat Exchanger Network. Since the 1960s, heat exchanger network integration technology has attracted significant attention as a subproblem of process design [32]. The transformation of the heat exchanger network effectively avoids the problems of temperature crossover and reverse heat transfer [33]. In 1965, Hwa first proposed structural optimization of heat exchanger networks [34]. In 1970, Ponton and Nishia proposed a trial method [35]. Shen et al. and Linnhoff et al. $[36,37]$ proposed the pinch analysis method, which is a method to solve the problem of inconsistency of energy flow in heat exchanger networks [38].

\section{Comparison of Simulation Results between the Actual and Optimized Processes}

3.1. Overview of Actual On-Site Processes. The purpose of process simulation is to realize an optimal design, operation, and modification of the model or system in a relatively short time by changing the relevant parameters in the model. The PR (Peng-Robinson) equation suitable for oil and gas processing units is chosen as the state equation for simulation. The natural gas components are shown in Table 1.

The process simulation model in Aspen Plus is depicted in Figure 1.

\subsection{Optimization Results}

3.2.1. Selection of the Deacidification Reagent. The original process is optimized step by step. Firstly, the deacidification reagent is selected, and the reagent type is changed at flow 134 for simulation. In the first simulation, the original site solution is added to only $3 \mathrm{~mol} / \mathrm{L} \mathrm{MDEA}$ solution. In the second simulation, $2 \mathrm{~mol} / \mathrm{L}$ MDEA and sulfolane are added to the compound amine solution with a molar ratio of $10: 3$. In the third simulation, $3 \% \mathrm{PZ}$ is added in $2 \mathrm{~mol} / \mathrm{L} \mathrm{MDEA}$ solution. A comparison of the results is shown in Table 2.

It can be easily seen that the purification effect of the activated MDEA solution is slightly better than that of the compound amine solution and it achieves the purpose of deep 


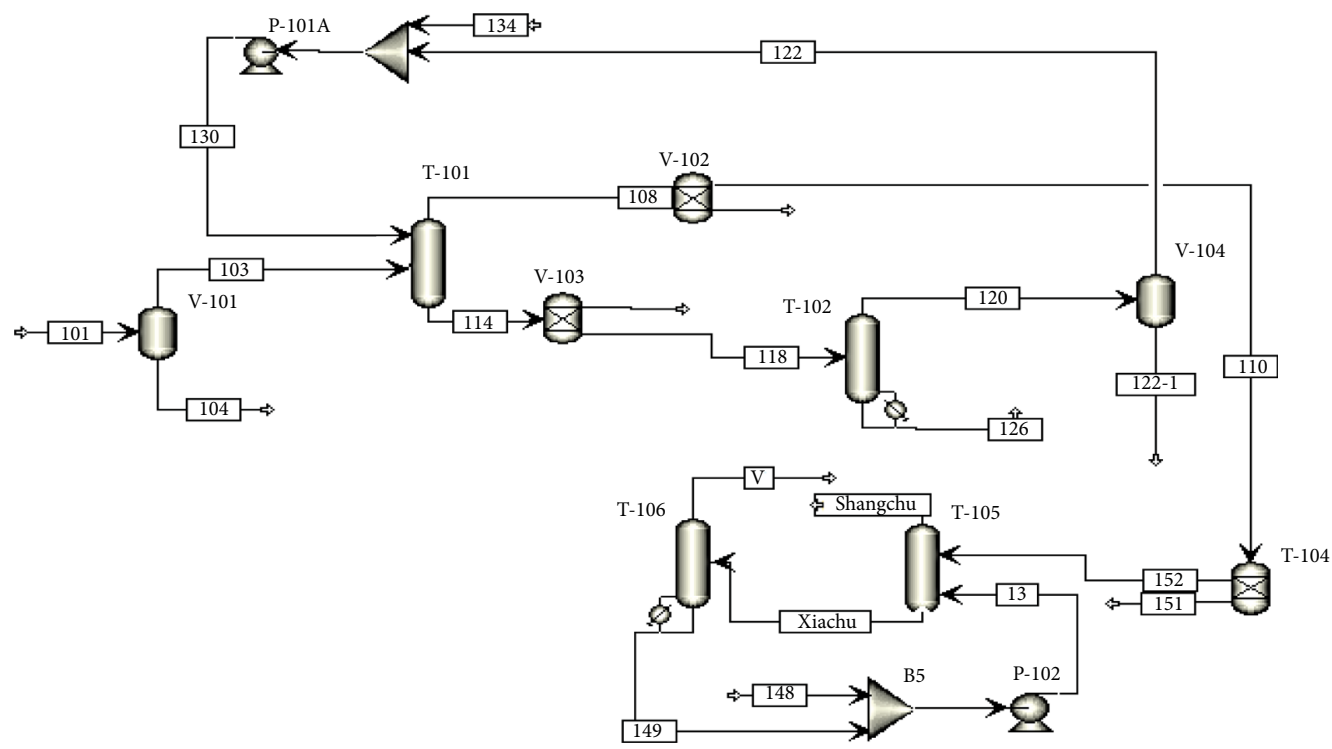

Figure 1: Actual process.

TABLe 2: Purification results with different reagents.

\begin{tabular}{lccc}
\hline Methods & MDEA solutions & Compounded MDEA solutions & Activated MDEA solutions \\
\hline Postpurification $\mathrm{CO}_{2}$ content $(\mathrm{mol} \%)$ & 0.0041 (the field observation values) & 0.0023 & 0.0018 \\
\hline
\end{tabular}

TABLE 3: Simple calculation results of the absorption tower.

\begin{tabular}{lc}
\hline Items & Value \\
\hline Absorption pressure & $5720(\mathrm{kPa})$ \\
Absorption temperature & $35\left({ }^{\circ} \mathrm{C}\right)$ \\
Gas-liquid ratio & $423\left(\mathrm{~m}^{3} / \mathrm{m}^{3}\right)$ \\
Number of actual stages & 15.3522 \\
\hline
\end{tabular}

purification, which is more amenable to the follow-up LNG processing process. Hence, this method is recommended for further analyses.

3.2.2. Absorption Tower Parameters. Simple calculation results of the absorption tower are shown in Table 3.

3.2.3. Semilean Liquid Process. To reduce the circulation of the absorption tower and the regeneration temperature of the regeneration tower, the optimized process uses a semilean solution scheme, as shown in Figure 2. To determine the entrance position of the semilean liquid, the number of trays can be increased appropriately to find out the rule. Therefore, sensitivity analysis of the absorption tower is carried out as shown in Figure 3. The $Y$ axis represents the gas phase molar fraction of $\mathrm{CO}_{2}$ and $\mathrm{CH}_{4}$ at different positions in the absorber, and the $X$ axis represents the number of theoretical trays (the first stage at the top of the absorber). By analyzing the distribution curves of key components in the absorber, it can be seen that the absolute values of the slope of $\mathrm{CO}_{2}$ and $\mathrm{CH}_{4}$ curves begin to decrease at $3 / 4$ and $1 / 3$ of the distance from the bottom of the absorber, indicating that

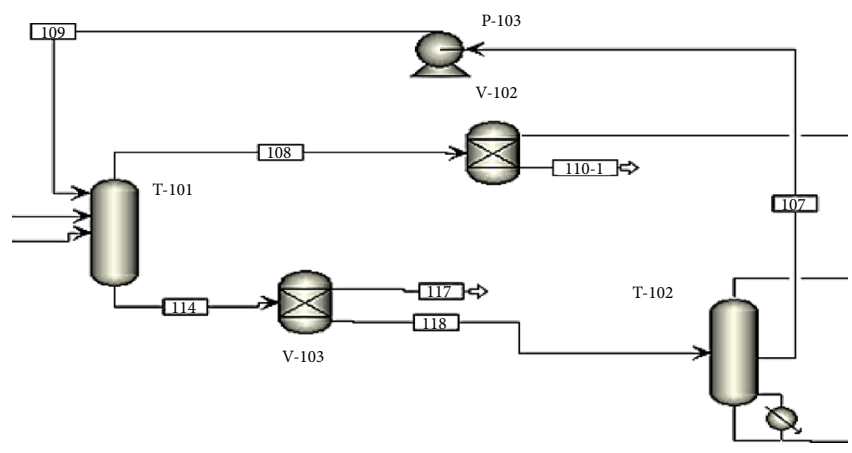

Figure 2: Schematic for the simulation of the semilean liquid process.

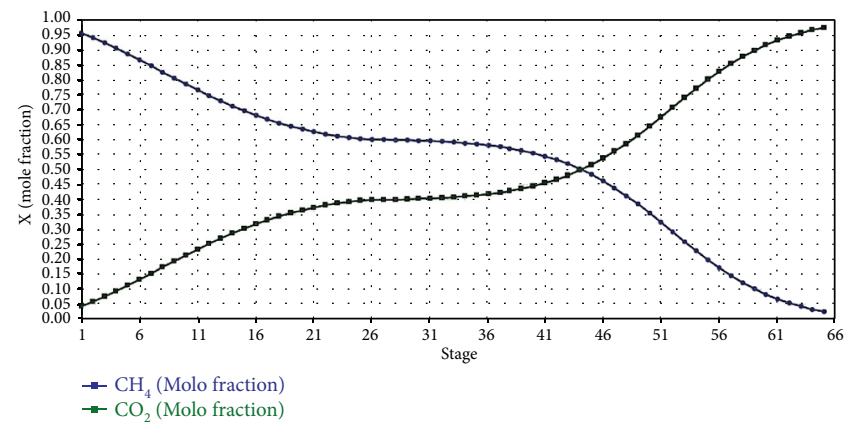

FIGURE 3: Distribution curve of the tower's key components. 


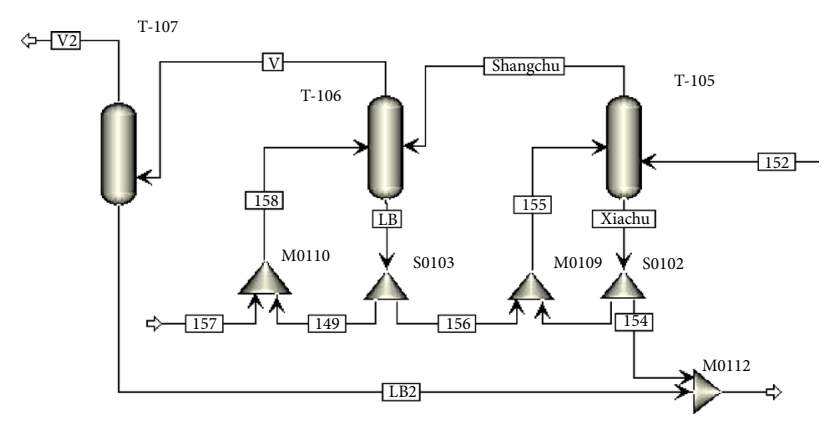

Figure 4: Process of three-tower dehydration.

the absorption efficiency decreases here. To maintain high absorption efficiency for a longer duration, it is recommended to introduce semilean liquid at $3 / 4$ or $1 / 3$ of the whole column.

3.2.4. Three-Tower Molecular Sieve Dehydration Process. In the traditional two-tower molecular sieve dewatering process, tower $\mathrm{A}$ is for the adsorption operation and tower $\mathrm{B}$ is for the regeneration of adsorbents. However, in the threetower and multitower processes, owing to various factors, switching procedures between different towers can be selected. Taking a three-tower process as an example, towers $\mathrm{A}, \mathrm{B}$, and $\mathrm{C}$ can be used for adsorption, regeneration, and cooling, respectively. Alternatively, towers A, B, and C can also be used for regeneration, adsorption, and cooling, respectively. Irrespective of these choices, the basic principles remain the same. The three-tower molecular sieve dewatering process has strong independence. It can not only save power but also eliminate a set of regenerated gas turbocharging units. A typical three-tower process is shown in Figure 4.

3.2.5. Optimization of the Heat Exchanger Network. Aspen Energy Analyzer is used to analyze the relationship between the operational cost, equipment cost index, and minimum heat transfer temperature difference. Considering the actual heat transfer effect and the goal of minimizing the total investment, the pinch point method is used to analyze the minimum heat transfer temperature difference of this project, which is $13^{\circ} \mathrm{C}$, as shown in Figure 5 .

Based on the premise of the minimum heat transfer temperature difference, the heat exchanger network is optimized. The specific optimization details are shown in Figure 6.

Specific optimization steps are as follows: the acid gas at the top of the amine liquid regeneration tower carries a large amount of latent heat at low temperatures, which cannot be directly used. Heat pump technology can be used to raise the acid temperature level and then be used as the heating heat source of the bottom reboiler to improve the heat transfer efficiency. The inlet gas matches the temperature and the heat load of the refrigeration cycle cooler perfectly during pressure swing adsorption, giving priority to the heat transfer. The outlet logistic temperature changes for a long time during the temperature swing adsorption operation, thus avoiding the use of process logistic heat transfer but using the circulating water for cooling. Similarly, the heater should

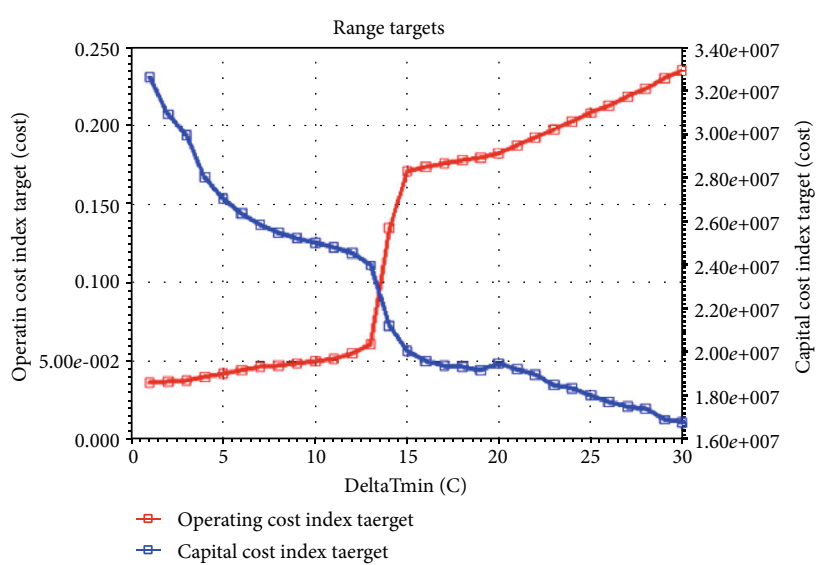

FIGURE 5: Minimum temperature difference diagram of heat transfer.

adopt steam heating to ensure the stability of the device. Through the optimization of the heat exchanger network, a total of nine heat exchanger flows are realized, and all the parts that need to be optimized are reflected in the simulation process. The process is transformed and constructed according to the optimization results. The improved simulation process is shown in Figure 7.

3.2.6. Exergy Analysis. The main components of the purification plant are the MDEA solution circulation system, triethylene glycol circulation system, production and fire water supply system, air-nitrogen system, boiler and boiler water supply system, sewage treatment system, and circulating water system. By comparing the energy consumption data of the components, it is found that the dominant power consumption components are the circulating pump of the MDEA circulating system and the air compressor of the air-nitrogen system. The power consumption details of these units are shown in Table 4.

The formula for the enthalpy loss in the heat exchanger is as follows, wherein 1 and 2 are the states before and after the heat exchange.

$$
\Delta E_{x}=\left(H-H_{0}\right)-T_{0}\left(S-S_{0}\right)+w_{c}
$$

where $T_{0}$ is the ambient temperature, ${ }^{\circ} \mathrm{C} ; H$ is the specific enthalpy, $\mathrm{kJ} ; H_{0}$ is the specific enthalpy under ambient temperature, $\mathrm{kJ} ; S$ is the entropy, kJ; $S_{0}$ is the entropy under ambient temperature, $\mathrm{kJ}$; and $w_{\mathrm{c}}$ is the compressor power consumption, $\mathrm{kW}$.

The formulae for calculating the rate loss in the heat exchangers are as follows, 1 and 2 of which are pre- and postheat transfer states.

$$
\Delta E_{x}=\sum E_{x, \text { in }}-\sum E_{x, \text { out }},
$$

where $E_{x \text {,in }}$ is the exergy entering the heat exchanger, $\mathrm{kJ}$; and $E_{x, \text { out }}$ is the exergy leaving the heat exchanger, $\mathrm{kJ}$. 


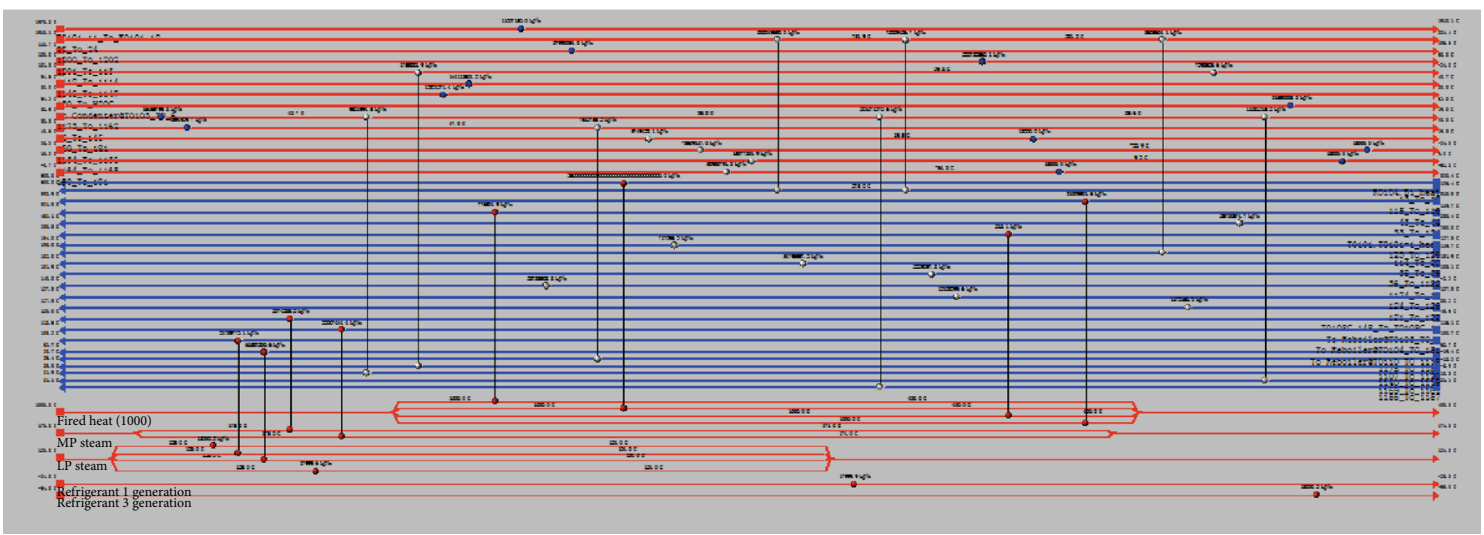

Figure 6: Optimized network of heat exchangers.

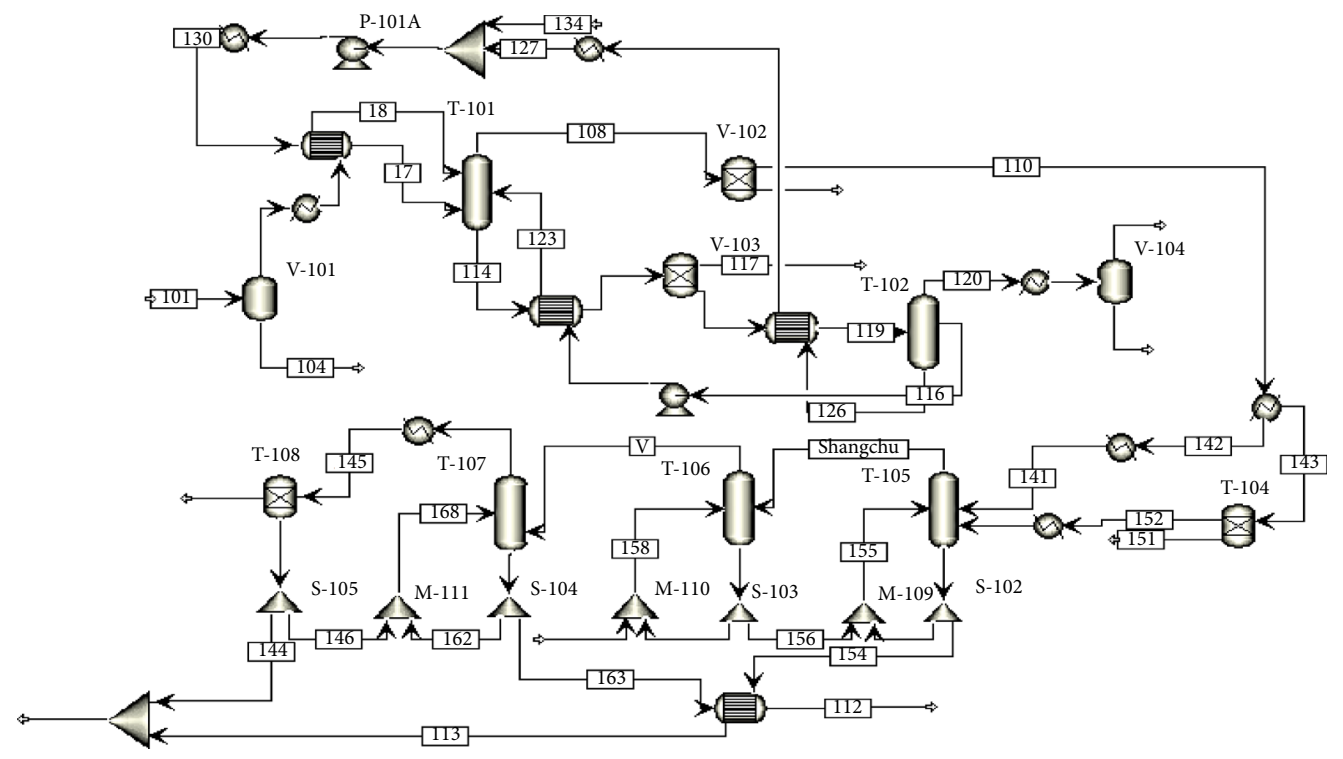

FIgURE 7: Optimized process.

According to the process flow of the device and considering the main energy-consuming process equipment, the model for the analysis is established as shown in Figure 8. The results of each logistic point in the plant under two operating conditions are shown in Table 5. Because the purpose of this study is to provide a basis for decision-making for the operation of the plant, additional fuel and other factors are needed in the analysis and calculation. The equations of equilibrium are shown as follows.

$$
\begin{gathered}
E_{1} c_{1}+E_{4} c_{4}=E_{2} c_{2}+E_{3} c_{3}, \\
E_{3} c_{3}+E_{5} c_{5}+E_{11} c_{11}+E_{12} c_{12}=E_{4} c_{4}+E_{6} c_{6}+E_{14} c_{14}, \\
E_{6} c_{6}+E_{8} c_{8}+E_{10} c_{10}=E_{5} c_{5}+E_{7} c_{7}+E_{9} c_{9}, \\
E_{7} c_{7}+E_{9} c_{9}+E_{14} c_{14}=E_{8} c_{8}+E_{10} c_{10}+E_{13} c_{13} \\
+E_{15} c_{15}+E_{18} c_{18}, \\
E_{15} c_{15}=E_{17} c_{17}+E_{18} c_{18},
\end{gathered}
$$

where $\mathrm{E}_{x}$ is the exergy value of $x, \mathrm{~kJ}$; and $c_{x}$ is the unit cost of $x, \mathrm{RMB}$ yuan/kJ.

There are a total of 18 unknowns and only 5 economic equilibrium equations. To solve the equations, auxiliary equations are established as follows:

(1) The unit costs of the inlet and outlet of the compression unit and the dry gas of the outlet are equal, i.e., $c_{6}=c_{7}=c_{14}$

(2) The unit costs of the terminal products are equal, i.e., $c_{17}=c_{18}$

(3) The raw material gas is close to the environmental state, and its unit cost is zero, i.e., $c_{12}=0$

(4) Fuel gas physical exergy is neglected. The gas used in the fuel gas system comes from a part of the produced gas of the well plant, and it is converted into atmospheric gas for combustion after passing through the throttle needle valve and pressure-reducing valve, and the temperature is normal temperature. 
TABLE 4: Energy consumption (power consumption) details of the circulating pump and the compressor.

\begin{tabular}{lccc}
\hline Description & Transmission medium & Voltage (V) & Power $(\mathrm{kW})$ \\
\hline Amine liquid circulating pump (I) & MDEA solutions & 380 & 90 \\
Amine liquid circulating pump (II) & MDEA solutions & 380 & 55 \\
The main air blower & Air & 380 & 132 \\
The screw air compressor & Air & 380 & 37 \\
\hline
\end{tabular}

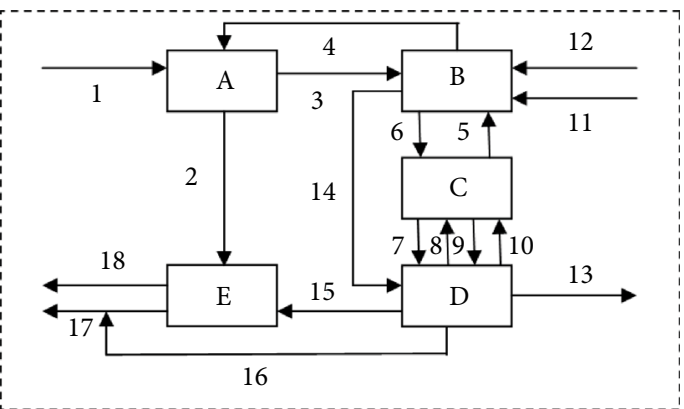

Figure 8: Analysis model of the process. A: regeneration unit; B: absorption unit; $C$ : heat exchange unit; D: drying unit; $E$ : compression unit.

TABLE 5: Analysis result of the process's exergy.

\begin{tabular}{lcc}
\hline $\begin{array}{l}\text { Condition } \\
\text { The nodes }\end{array}$ & $\begin{array}{c}\text { The actual values (I) } \\
\text { Exergy values, }\end{array}$ & $\begin{array}{c}\text { The optimized values (II) } \\
\mathrm{kJ} / \mathrm{h}\end{array}$ \\
\hline 1 & 1.33 & 5.19 \\
2 & 152.69 & 131.74 \\
3 & 751.67 & 1018.98 \\
4 & 1621.80 & 1787.10 \\
5 & 3.21 & - \\
6 & 1474.30 & 1863.31 \\
7 & 503.90 & 697.88 \\
8 & 660.75 & 834.34 \\
9 & 223.94 & 333.45 \\
10 & 381.83 & 615.41 \\
11 & 5993.28 & 6374.88 \\
12 & 42.55 & 128.58 \\
13 & 283.06 & 370.38 \\
14 & 0.81 & - \\
15 & 97.66 & 87.83 \\
16 & 534.82 & 799.98 \\
17 & 41.37 & 20.18 \\
18 & 229.38 & 349.05 \\
\hline & &
\end{tabular}

Therefore, in order to make the calculation simple and the equation have a unique solution, it is ignored

(5) The unit cost of dry gas is calculated according to the following formula:

$$
c=\frac{c_{13} \times E_{13}+\left(Q-Q_{13}\right) \times e_{17} \times c_{17}}{E},
$$

where $Q$ is the flow of dry gas, $\mathrm{m}^{3} ; Q_{13}$ is the flow of node 13 , $\mathrm{m}^{3}$; and $e_{17}$ is the specific energy of node 17 .

The enthalpy loss of the compression process in the original process flow is calculated to be $32 \mathrm{MJ} / \mathrm{h}$, and the enthalpy loss in the heat exchange process is $46.2 \mathrm{MJ} / \mathrm{h}$. After optimization, the process enthalpy loss is $29.04 \mathrm{MJ} / \mathrm{h}$ and the heat exchange enthalpy loss is $42.715 \mathrm{MJ} / \mathrm{h}$. This translates to an increase of efficiency by approximately $9 \%$. Thus, the expected energy-saving goals have been achieved.

\section{Conclusion}

(1) As per the data from the single-factor analysis and the Aspen Plus simulation, the purification rate of $\mathrm{CO}_{2}$ by MDEA with $\mathrm{PZ}$ as an activator reached $99.94 \%$, which is significantly higher than that by the MDEA-sulfolane mixed solution method. While ensuring the purification effect, the semilean ammonia liquid circulation process reduced the ammonia liquid circulation by more than $49 \%$ compared with the ordinary lean/rich ammonia liquid circulation process. Furthermore, this also reduced the regeneration load of the ammonia liquid regeneration tower and achieved the goal of energy saving. Using a three-tower molecular sieve in the optimized dehydration process can not only avoid the investment cost caused by the TEG loss but also save 583,500 yuan per year for the plants based on a consumption of $14.03 \mathrm{mg}$ of triglyceride per $\mathrm{m}^{3}$ of natural gas treatment. At the same time, it can meet the temperature requirements of downstream LNG production

(2) Based on the analysis of the heat exchanger network, the pinch analysis method is used to optimize the heat exchanger network of the optimized purification process. From the thermodynamic point of view, the optimal matching of the heat exchanger logistics and the thermodynamic goal of minimum energy consumption are achieved. In addition, the problem of inconsistency of energy flow in the heat exchanger network is solved. Finally, the efficiency of the system is improved. Thus, all the goals of optimization have been achieved

\section{Data Availability}

The data used to support the findings of this study are available within the article. 


\section{Conflicts of Interest}

The authors declare that they have no conflicts of interest.

\section{Acknowledgments}

The authors gratefully expressed their thanks for the financial support given by the National Natural Science Foundation of China (No. 51974033) and the Educational Commission of Hubei Province of China (D20171305).

\section{References}

[1] Y. Alcheikhhamdon and M. Hoorfar, "Natural gas quality enhancement: a review of the conventional treatment processes, and the industrial challenges facing emerging technologies," Journal of Natural Gas Science and Engineering, vol. 34, pp. 689-701, 2016.

[2] L. Jiangang, Study on removal of acid gas (H2S, CO2) and its gas-liquid mass transfer characteristics, Nanjing University of Since and Technology, Nanjing, 2005.

[3] W. Xue and Z. Zeng, "Study on natural gas purification membrane and gas mass transfer mechanism in membrane," Journal of Chemical Engineering of Chinese Universities, vol. 13, no. 5, pp. 408-414, 1999.

[4] T. E. Rufford, S. Smart, G. C. Y. Watson et al., "The removal of $\mathrm{CO} 2$ and N2 from natural gas: a review of conventional and emerging process technologies," Journal of Petroleum Science and Engineering, vol. 94-95, pp. 123-154, 2012.

[5] J. K. Adewole, A. L. Ahmad, S. Ismail, and C. P. Leo, "Current challenges in membrane separation of $\mathrm{CO} 2$ from natural gas: a review," International Journal of Greenhouse Gas Control, vol. 17, pp. 46-65, 2013.

[6] J. A. Lagas and M. Pang, "Removal of acid gas by selective amine process," Chemical Engineering of Oil \& Gas, vol. S1, pp. 60-66, 1983.

[7] J. Liu, Study on natural gas recovery technology in outlying wells, Southwest Petroleum University, Chengdu, 2016.

[8] M. Yao, Experimental study on CO2 absorption-desorption process based on organic amine method and energy consumption analysis of capture system, Beijing Jiaotong University, Beijing, 2014.

[9] Y. Chen, Y. Yao, Z. Jiang et al., "Analysis of $\mathrm{CO}_{2}$ capture process in flue gas by mixed alkanolamines MEA-AMP," Computers and Applied Chemistry, vol. 29, no. 1, pp. 113-116, 2012.

[10] J. Li, Study on formulation screening of selective desulfurization amine solution for natural gas, China University of Petroleum, Qingdao, 2016.

[11] K. Wang, "Review od the development process of natural gas desulfurization and carbon removal technology-MDEA is now in an outstanding position," Natural Gas and Petroleum, vol. 29, no. 1, pp. 15-21, 2011.

[12] Y. Wang and D. Wang, "Selection of MDEA formula solution in desulfurization and carbon removal of natural gas," Chemical Engineering of Oil \& Gas, vol. 32, no. 5, pp. 291-294, 2003.

[13] R. Zheng, Study on natural gas purification and acid gas treatment technology with high C/S ratio, Southwest Petroleum University, Chengdu, 2014.

[14] L. Zhao, Simulation and optimization of natural gas purification process, Northeast Petroleum University, Daqing, 2012.
[15] N. Chen, Application research of activated MDEA decarbonization, Southwest Petroleum University, Chengdu, 2016.

[16] Y. Shi, Research and implementation of PZ activated MDEA decarbonization process model software based on multi-core SVR, Xi'an Shiyou University, Xi'an, 2018.

[17] Y. Meng and D. Qi, "Optimization of operation parameters of MDEA absorption tower," Oil-Gas field Surface Engineering, vol. 31, no. 7, pp. 22-23, 2012.

[18] J. Li, Z. Ye, J. Wu, Z. Fan, and Z. Wang, "Effective energy analysis and energy saving measures of alcohol amine natural gas desulfurization and carbon removal unit," Modern Chemical Industry, vol. 38, no. 6, pp. 186-191, 2018.

[19] H. Zhang, Design and application of natural gas liquefaction unit and technology, South China University of Technology, Guangzhou, 2014.

[20] Y. Guo, Research on technology and application of natural gas liquefaction, Xi'an Shiyou University, Xi'an, 2011.

[21] K. Dalane, Z. Dai, G. Mogseth, M. Hillestad, and L. Deng, "Potential applications of membrane separation for subsea natural gas processing: a review," Journal of Natural Gas Science and Engineering, vol. 39, pp. 101-117, 2017.

[22] C. Scholes, K. H. Smith, S. Kentish, and G. Stevens, "CO2 capture from pre-combustion processes-strategies for membrane gas separation," International Journal of Greenhouse Gas Control, vol. 4, no. 5, pp. 739-755, 2010.

[23] Q. Ye, Study on optimization of small and medium-sized natural gas liquefaction process, China University of Petroleum, 2016.

[24] L. Cheng, Study on factors affecting the life of TEG in natural gas dehydration process in Wanzhou operating area and countermeasures, Chongqing University of Science and Technology, Chongqing, 2017.

[25] M. Safari, A. Ghanizadeh, and M. Montazer-Rahmati, "Optimization of membrane-based $\mathrm{CO} 2$-removal from natural gas using simple models considering both pressure and temperature effects," International Journal of Greenhouse Gas Control, vol. 3, no. 1, pp. 3-10, 2009.

[26] X. Zhang, D. Wang, R. Liao, S. Wang, B. Shi, and L. Wu, "Associated petroleum gas measurement at low gas content using PIS method," Flow Measurement and Instrumentation, vol. 70, article 101662, 2019.

[27] Y. Liu, Study on optimization of natural gas ethane recovery process, Southwest Petroleum University, Chengdu, 2016.

[28] B. Tirandazi, M. Mehrpooya, A. Vatani, and S. M. A. Moosavian, "Exergy analysis of $\mathrm{C} 2+$ recovery plants refrigeration cycles," Chemical Engineering Research and Design, vol. 89, no. 6, pp. 676-689, 2011.

[29] X. Zhang, Z. Jiang, R. Liao et al., "Study on temperature distribution of perforated horizontal wellbore," Journal of Thermal Science, vol. 29, no. 1, pp. 194-205, 2020.

[30] J. Yang, Y. Ma, M. Li, and H. Guan, "Exergy analysis of transcritical carbon dioxide refrigeration cycle with an expander," Energy, vol. 30, no. 7, pp. 1162-1175, 2005.

[31] S. Zhang, Research and analysis of natural gas liquefaction process with mixed refrigerant refrigeration, Southwest Petroleum University, Chengdu, 2017.

[32] Y. Huang, Chemical process design: chemical engineering and technology, Xi'an Technology University Press, Xi'an, 2005.

[33] L. Din, Research on deep condensate recovery technology of natural gas in TLM gas field, Southwest Petroleum University, Chengdu, 2016. 
[34] T. Gundepsen and L. Naess, "The synthesis of cost optimal heat exchanger networks," Computers \& Chemical Engineering, vol. 12, no. 6, pp. 503-530, 1988.

[35] J. Wang, Chemical process design, Chemical Industry Press, Beijing, 2nd Ed. edition, 2006.

[36] Q. Shen, Y. Jiang, F. Xia et al., "Hydrogen production by cobased bimetallic nano-catalysts and their performance in methane steam reforming," Petroleum Science and Technology, vol. 38, no. 6, pp. 618-625, 2020.

[37] B. Linnhoff and E. Hindmarsh, "The pinch design method for heat exchanger networks," Chemical Engineering Science, vol. 38, no. 5, pp. 745-763, 1983.

[38] H. Zhou, Z. Wang, and Z. Yao, "Pinch point analysis and energy saving potential of Dongyilian," Contemporary Chemical Industry, vol. 44, no. 11, pp. 2659-2661, 2015. 